\title{
First-Principle Study on the Interaction between Fe and Trivacancy in Graphene
}

\author{
Xielong Hu and Fanyan Meng \\ Department of Physics, University of Science \& Technology Beijing, Beijing 100083, China \\ Correspondence should be addressed to Fanyan Meng; meng7707@sas.ustb.edu.cn
}

Received 30 October 2015; Revised 11 December 2015; Accepted 20 December 2015

Academic Editor: Shamsul Arafin

Copyright (C) 2016 X. Hu and F. Meng. This is an open access article distributed under the Creative Commons Attribution License, which permits unrestricted use, distribution, and reproduction in any medium, provided the original work is properly cited.

\begin{abstract}
$\mathrm{Ab}$ initio calculations using density functional theory (DFT) have been performed in order to explore structure and energy gap opening of graphene with bridged-trivacancy and single adsorbed with Fe atom. Compared to the previous reconstructed trivacancy adsorbed with $\mathrm{Fe}$ atom with the energy gap of $0.10 \mathrm{eV}$, one interesting structure for the Fe-doped bridged-trivacancy complex has been identified, with one Fe atom above the graphene plane, and possesses energy gap with the value of $0.32 \mathrm{eV}$ in the bridged circumstance. The band gap can be explained by the decrease of the free electrons. These results provide insights to engineer graphene's properties through defect addition and manipulation for industrial semiconductor applications such as the photocatalytic technology and graphene based electronics.
\end{abstract}

\section{Introduction}

Graphene is among the most potential utilized materials currently studied [1-3]. It exhibits extraordinary properties in electrology $[4,5]$, magnetism [6,7], and dynamics [8], proved by various theoretical and experimental studies. However, graphene is a semimetal, which greatly limits the application in the graphene-based electronic field [9-11]. Consequently, in the recent decades, many approaches, such as by employing a staggered $A B$ sublattice potential [12], strain effect [13], intrinsic spin-orbit coupling [14] in single-layer graphene, functionalization with chemical groups [15-17], or applying a perpendicular electric field in bilayer graphene [18], have been explored to open the gap of graphene for designing semiconductor device.

Chan et al. have studied the structures and magnetism about pure graphene adsorbed with many elements such as $\mathrm{Li}$, $\mathrm{Na}, \mathrm{K}, \mathrm{Ca}$, and $\mathrm{Al}[19]$. However, precise and stable structures of the transition metal (TM) adsorbed on graphene are hardly obtained because TM adsorbents can move freely on the surface of pristine graphene, which limits the use of surface adatom as robust and reliable dopants for nanoelectronic and magnetic applications [20]. Recently, it is forecasted that attaching metal atoms to defected graphene is much firmer than to pristine graphene by theoretical studies [21-25]. Kim et al. have used X-ray magnetic circular dichroism (XMCD) to study the electronic and magnetic properties of transition metal (TM), for example, Fe, Co, and Ni clusters on monolayer graphene [26]. Also a very high magnetic anisotropy energy (MAE) has been found in single-vacancy graphene adsorbed with Ir dimer [27]. Dynamics of single Fe adatom on graphene vacancies have been studied using focused electron beam irradiation [28]. Vacancy-assisted doping method is one available technique for introducing dopants into graphene.

As a matter of fact, iron in graphene nanostructures are very common in experiment, such as Fe pairs doped in graphene [20], single Fe atoms in graphene vacancies [29,30], and $\mathrm{Fe}_{3} \mathrm{O}_{4}$ /graphene nanocomposites [31-34]. In previous work, band gap of graphene could be opening via Fe atom adsorption [11]. Recently, an interesting structure of graphene trivacancy, which was achieved by using an electron beam to bombard the graphene sheet, has been commonly observed by Robertson et al. [35]. The new structure has a bridging atom (thereafter called bridged-trivacancy), significantly different from the reconstructed trivacancy ( $r$-trivacancy) observed by Wang et al. [36]. Consequently, a combination 


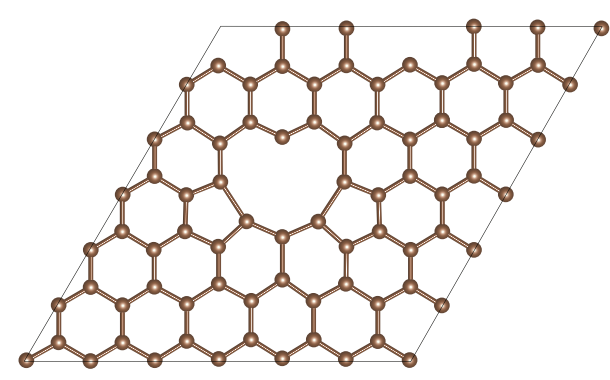

(a)

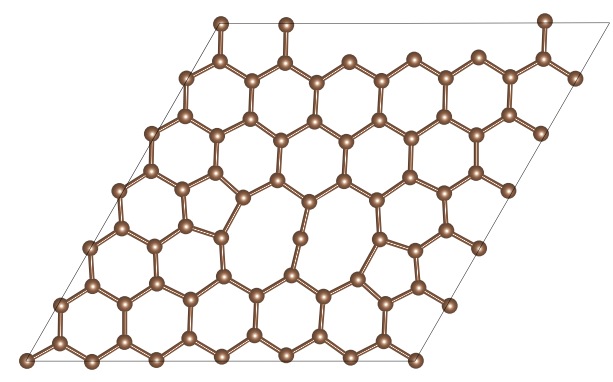

(b)

Figure 1: (a) Optimized $6 \times 6$ graphene supercell with $\mathrm{r}$-trivacancy. (b) Optimized $6 \times 6$ graphene supercell with b-trivacancy.

of Fe adsorption and b-trivacancy might contribute to extend the application of graphene by opening the band gap.

This work presents a detailed study of the structure and electronic properties of graphene with r-trivacancy and btrivacancies trapping $\mathrm{Fe}$ atom, using the density functional theory (DFT). Our studies may verify the feasibility of improving graphene electrical properties by controlling TM atom doping and vacancies.

\section{Calculation Details}

First-principles calculations based on the spin-polarized density functional theory (DFT) were performed using the projector augmented wave (PAW) [37], as implemented in the Vienna Ab initio Simulation Package (VASP) program [38]. The generalized gradient approximation in the PerdewBurke-Ernzerhof (PBE) [39] parameterization was employed for the exchange-correlation functional. The plane-wave cutoff energy was set to $500 \mathrm{eV}$. The geometry optimization and total energy calculations were performed until the residual force was within $0.01 \mathrm{eV} / \AA$ and the total energy converged to $10^{-5} \mathrm{eV}$. The $k$-point mesh for sampling the Brillouin zone was generated including the gamma point. A $4 \times 4 \times 1 k$-point mesh was used for geometry optimization, and a $6 \times 6 \times 1$ $k$-point mesh was used for total energy calculations.

We modeled the defect systems using supercells large enough $(5 \times 5$ and $6 \times 6$ supercells in the lateral direction with a $20 \AA$ thick vacuum in the vertical direction) to minimize boundary effects on the energetics of the systems. Graphene's lattice constant was calculated to be $2.470 \AA$, which is similar to the calculated value $(2.468 \AA)$ by Dai et al. and the experimental value of $2.46 \AA$ [21].

\section{Structures for System}

Three kinds of trivacancies in graphene have been discovered in previous research, the r-trivacancy observed by Wang in 2012 [35] and the b-trivacancy observed by Robertson in 2014 [36], and the optimized equilibrium configurations for the r-trivacancy and b-trivacancy in our simulations using DFT are shown in Figure 1. The b-trivacancy is not just stabilized by simple bond reconstructions between undercoordinated carbon atoms, as exhibited by r-trivacancy. Reconstructions consist of undercoordinated bridging carbon atoms spanning the vacancy to saturate edge atoms in b-trivacancy, which matches well with the previous work [36].

All the systems with $\mathrm{Fe}$ adsorbate on perfect graphene and defective graphene (containing a r-trivacancy or btrivacancy) are studied. Three possible adsorption sites of Fe on the perfect graphene are labeled as follows: hollow $(\mathrm{H})$, top (T), and bridge (B). And the complexes of perfect graphene adsorbed with Fe which locates in the three possible sites are indicated as Fe@G(H), Fe@G(T), and Fe@G(B), respectively. For adsorption on defect-free graphene, Fe@G(H) (on the hollow site) is the most stable complex, which is in good agreement with the previous theoretical results $[5,19]$. In the case of the adsorption of Fe on the defective graphene, $\mathrm{Fe}$ is positioned at different hollow, top, and bridge sites in the trivacancy region as the initial positions and then fully relaxed. The configurations of the adsorption of Fe on defective graphene are shown in Figure 2, in both top view and side view. For convenience, the complex of Fe adsorbed on the graphene with a r-trivacancy is labeled as Fe@RTV, and the one with b-trivacancy as Fe@BTV. From the side views, it is obvious that $\mathrm{Fe}$ atom is above the plane of graphene with btrivacancy and the adatom-graphene distance $d(\AA)$ is $1.38 \AA$.

The formation energy $E_{f}$ of a trivacancy in graphene, indicated by $E_{f}$, is defined as

$$
E_{f}=E_{s}-\frac{N-n}{N} E_{g}
$$

where $n=3$ is the number of vacant $\mathrm{C}$ atoms in the system, and $N$ is the total number of $C$ atoms in supercells of the perfect graphene. $E_{s}$ and $E_{g}$ are the energies of the graphene with r-trivacancy or b-trivacancy and the $5 \times 5$ or $6 \times 6$ graphene supercells, respectively. The adsorption energy of Fe on the perfect or defective graphene is defined as

$$
E_{\mathrm{ads}}=E_{s}-E_{g}-E_{\mathrm{Fe}},
$$

where $E_{s}, E_{g}$, and $E_{\mathrm{Fe}}$ are the energies for the optimized systems of Fe-graphene with or without trivacancy, the $5 \times 5$ or $6 \times 6$ graphene supercells with or without trivacancy, and the isolated Fe atom, respectively.

Table 1 summarizes the energetic and structural properties of the $5 \times 5$ and $6 \times 6$ supercells of graphene with trivacancy adsorbed with Fe. For comparison, the formation energy of the trivacancy in graphene and the adsorption 
TABLE 1: Energetic and structural properties of Fe on the defect-free graphene and defective graphene with r-trivacancy and b-trivacancy. The properties listed include the size of graphene supercells $s$, types of the Fe-graphene complex $t$, the Fe-graphene distance $d$ ( $\AA$ ), formation energy $E_{f}(\mathrm{eV})$ of trivacancy in graphene, adsorption energies $E_{\mathrm{ads}}(\mathrm{eV})$, bulk band gap $\Delta(\mathrm{eV})$, and the magnetization of the adatom $\mu_{\mathrm{ag}}\left(\mu_{\mathrm{B}}\right)$. For comparison, the magnetization of the graphene with a trivacancy is included.

\begin{tabular}{|c|c|c|c|c|c|c|}
\hline$s$ & $t$ & $d(\AA)$ & $E_{f}(\mathrm{eV})$ & $E_{\text {ads }}(\mathrm{eV})$ & $\Delta(\mathrm{eV})$ & $\mu_{\mathrm{ag}}\left(\mu_{\mathrm{B}}\right)$ \\
\hline \multirow{7}{*}{$5 \times 5$} & Fe@RTV & 0.00 & - & -6.35 & 0.13 & 2.00 \\
\hline & Fe@BTV & 1.24 & - & -6.90 & 0.32 & 0.00 \\
\hline & $\mathrm{Fe} @ \mathrm{G}(\mathrm{H})$ & 1.65 & - & -1.04 & 0.08 & 2.00 \\
\hline & $\mathrm{Fe} @ \mathrm{G}(\mathrm{B})$ & 2.23 & - & -0.28 & 0.00 & 4.10 \\
\hline & $\mathrm{Fe} @ \mathrm{G}(\mathrm{T})$ & 2.06 & - & -0.27 & 0.00 & 4.10 \\
\hline & r-trivacancy & - & 11.68 & & 0.00 & 1.05 \\
\hline & b-trivacancy & - & 14.86 & & 0.00 & 1.66 \\
\hline \multirow{7}{*}{$6 \times 6$} & Fe@RTV & 0.00 & - & -6.61 & 0.03 & 2.00 \\
\hline & Fe@BTV & 1.38 & - & -6.96 & 0.25 & 0.00 \\
\hline & $\mathrm{Fe} @ \mathrm{G}(\mathrm{H})$ & 1.50 & - & -1.13 & 0.07 & 2.00 \\
\hline & $\mathrm{Fe} @ \mathrm{G}(\mathrm{B})$ & 2.20 & - & -0.29 & 0.00 & 4.10 \\
\hline & $\mathrm{Fe} @ \mathrm{G}(\mathrm{T})$ & 2.12 & - & -0.28 & 0.00 & 4.10 \\
\hline & r-trivacancy & - & 11.13 & & 0.00 & 1.05 \\
\hline & b-trivacancy & - & 14.27 & & 0.00 & 1.66 \\
\hline
\end{tabular}

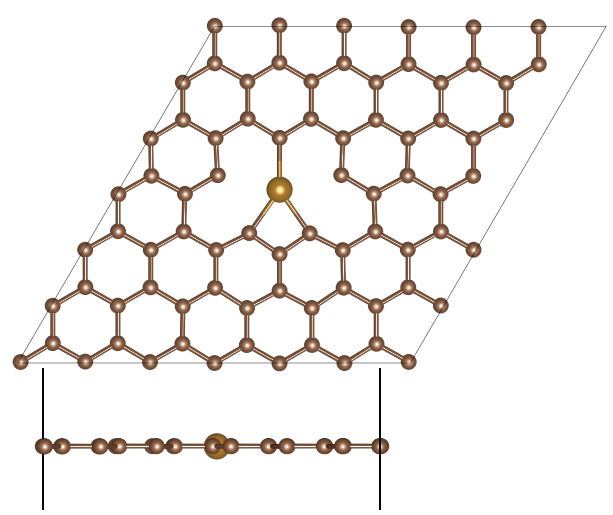

(a)

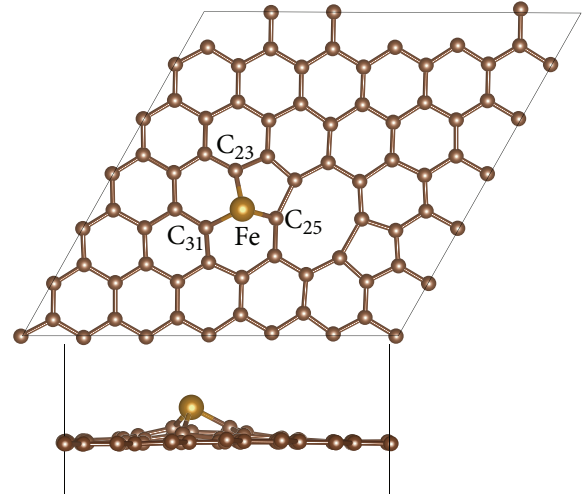

(b)

FIGURE 2: The optimized configurations of Fe adsorbed on the $6 \times 6$ graphene supercell with a trivacancy in both top view and side view. (a) Fe on the graphene with r-trivacancy (Fe@RTV) and (b) Fe on the graphene with b-trivacancy (Fe@BTV).

energy of Fe on the defect-free graphene are also included in Table 1. The adsorption energy for Fe@G(H) complex is the lowest, indicating that the stable position is the hollow site. The adsorption energy of Fe adsorbed on defective graphene with a trivacancy (Fe@TV) is much lower than that of adsorption on perfect graphene, indicating Fe@TV is more stable, which conforms to the previous studies [22]. It means that the introduction of a trivacancy on graphene will improve the adsorption of $\mathrm{Fe}$ atom. The formation energy of r-trivacancy is $11.68 \mathrm{eV}$, consistent with the result in [21], lower than that of b-trivacancy $(14.86 \mathrm{eV})$, indicating that the b-trivacancy is a metastable structure. It is in good agreement with previous studies [35]. From Table 1, we also notice that graphene supercell with b-trivacancy adsorbed with Fe (Fe@BTV) has a lower adsorption energy in comparison with r-trivacancy adsorbed with Fe (Fe@RTV) in the same concentration, which relates with a better incorporation of the adsorbate into the graphene framework. It is worth noting that the band gaps of Fe@BTV are $0.32 \mathrm{eV}$ and $0.25 \mathrm{eV}$ for $5 \times 5$ and $6 \times 6$ graphene supercells, respectively. By contrast, the band gaps of Fe@RTV are $0.13 \mathrm{eV}$ and $0.03 \mathrm{eV}$ for $5 \times 5$ and $6 \times 6$ supercells, respectively. The system of Fe adsorbed on the perfect graphene has the maximum band gaps of $0.08 \mathrm{eV}$, which is similar to the result (100.8 meV) in [11]. Fe@BTV system may have a bearing on important applications such as graphenebased electronic device.

\section{DOS for Fe@BTV}

The graphene with b-trivacancy and r-trivacancy is metallic, as the existence of trivacancy in graphene will increase the number of free electrons in the system. The partial densities of states (PDOS) for spin-up and spin-down electrons of Fe atom and adjacent $\mathrm{C}$ atoms and the total density of states (TDOS) in $5 \times 5$ and $6 \times 6$ graphene are calculated as shown in Figure 3. The indices of $\mathrm{C}$ atoms are labeled in Figure 2(b) 

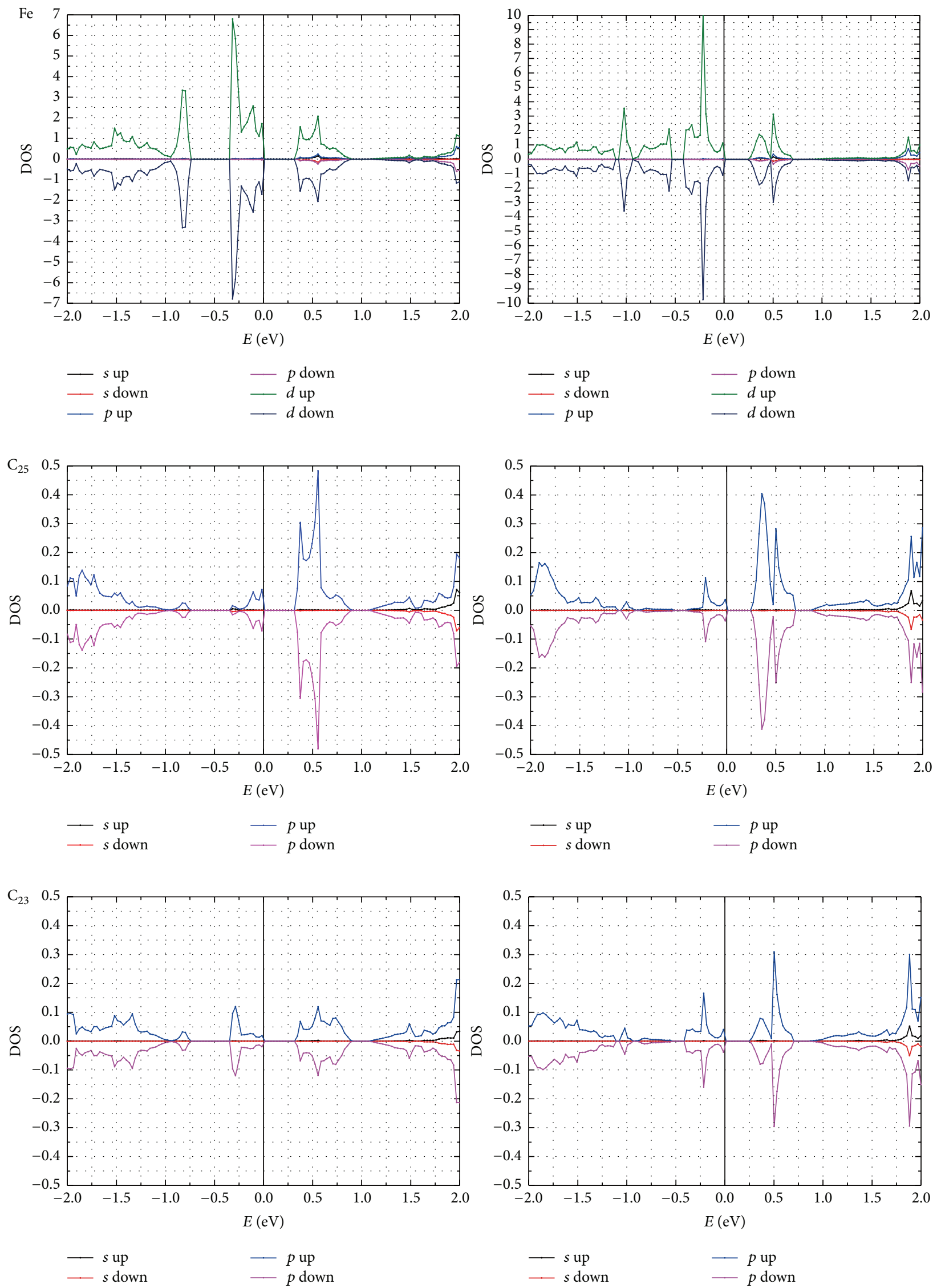

Figure 3: Continued. 

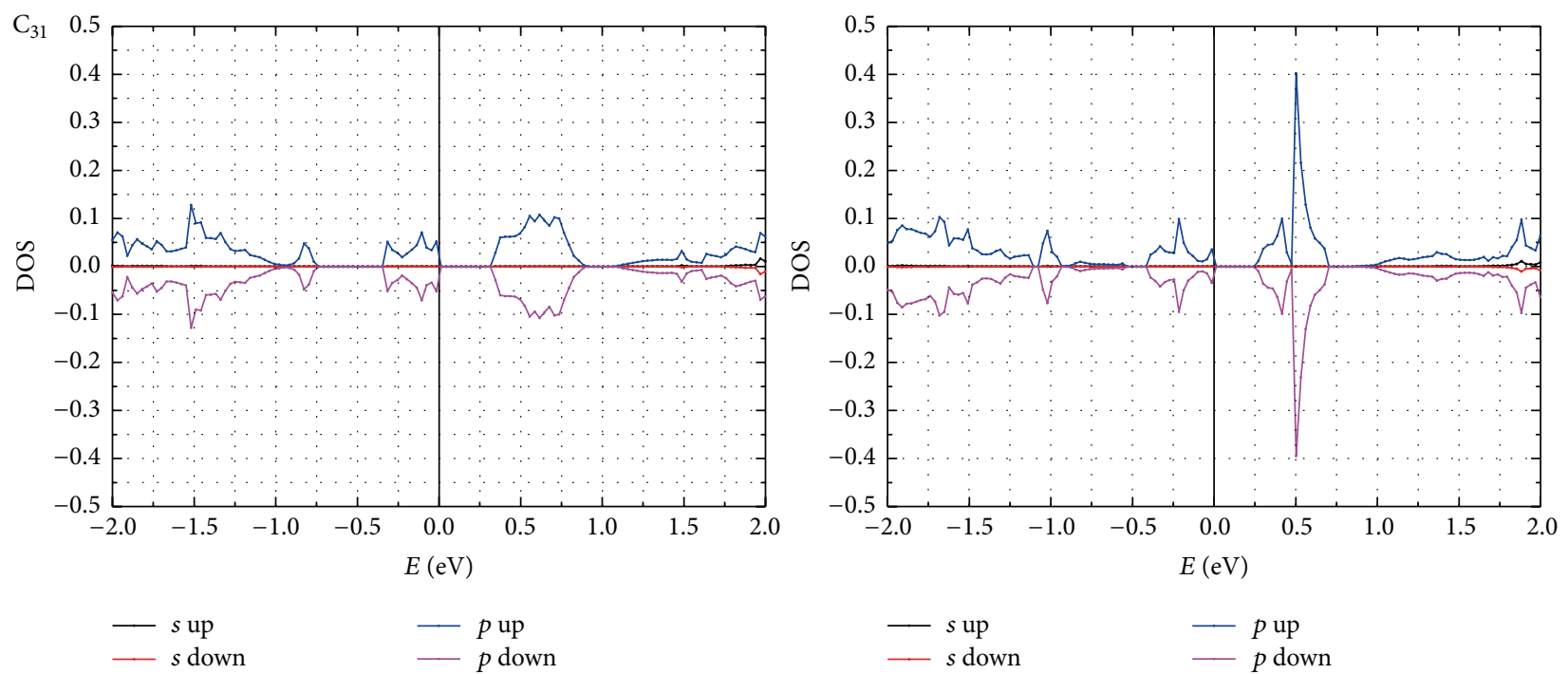

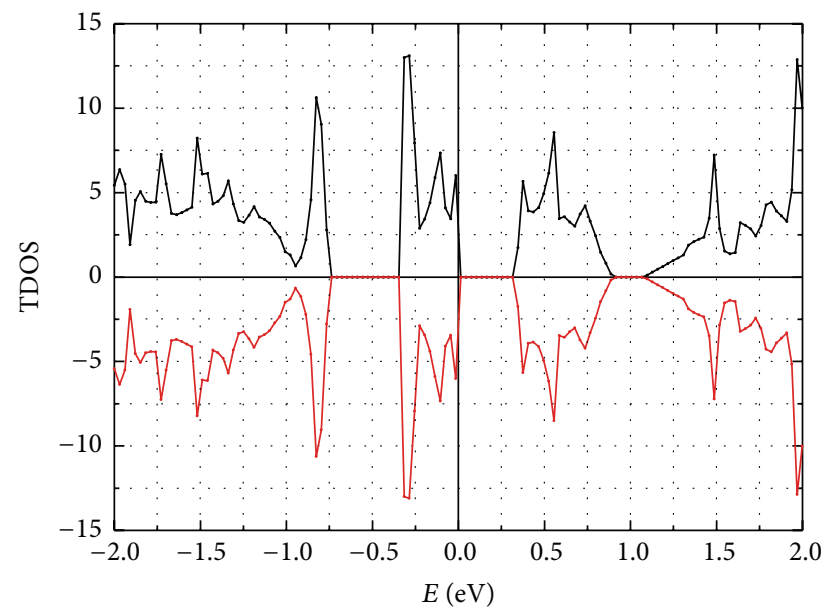

(a)

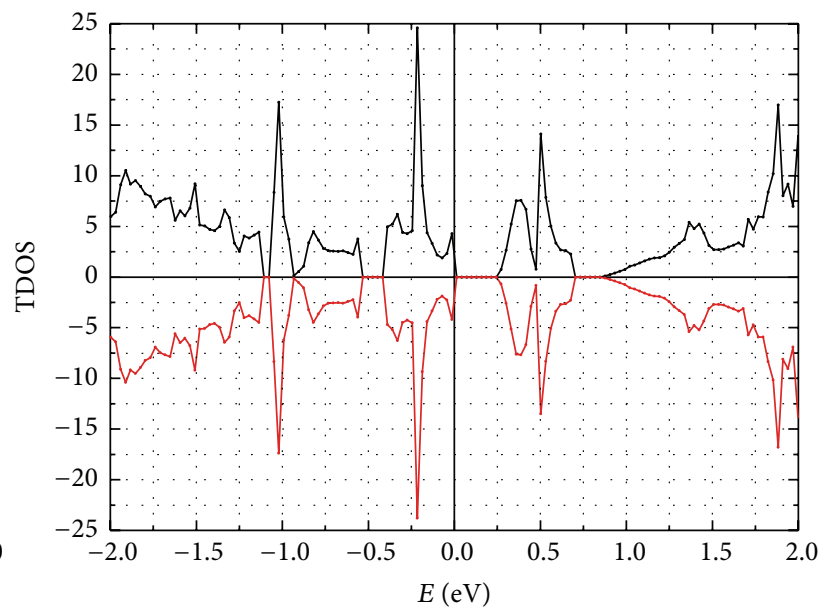

(b)

FIgURE 3: The partial densities of states (PDOS) for spin-up and spin-down electrons for Fe atom and C atoms vicinity of adsorbed Fe and the total density of states (TDOS) in $5 \times 5$ and $6 \times 6$ graphene: (a) $5 \times 5$ graphene, (b) $6 \times 6$ graphene.

and $\mathrm{C}_{25}$ labels the bridge $\mathrm{C}$ atom. Obviously, for b-trivacancy, the spin-up and spin-down DOS of $\mathrm{C}_{25}, \mathrm{C}_{23}, \mathrm{C}_{31}, \mathrm{Fe}$, and Fe@BTV is mirror symmetry, indicating no magnetic moment. The PDOS of adjacent $\mathrm{C}$ atoms match well with $\mathrm{Fe}$ $3 d$ orbitals. In $5 \times 5$ graphene all the PDOS locate at $-2 \sim$ $-0.75 \mathrm{eV},-6 \sim 0 \mathrm{eV}$, and $0.32 \sim 0.7 \mathrm{eV}$. Similar conclusions can be got in $6 \times 6$ graphene. In particular, the PDOS of $\mathrm{C}_{25} 2 p$ orbitals match so consistently with $\mathrm{Fe} 3 d$ orbitals. It suggests that $\mathrm{Fe}$ atom has formed covalent bonds with adjacent $\mathrm{C}$ atoms. Stronger $\mathrm{Fe}-\mathrm{C}$ interaction was observed between $\mathrm{Fe}-$ $\mathrm{C}_{25}$ than that between $\mathrm{Fe}-\mathrm{C}_{23}$ and $\mathrm{Fe}-\mathrm{C}_{31}$. The magnetization of Fe in b-trivacancy is $0 \mu_{\mathrm{B}}$ while the Fe atom in r-trivacancy has magnetization of $2 \mu_{\mathrm{B}}$ (Table 1). This is consistent with the fact that there are enough ten electrons in the $3 d$ orbitals of Fe in b-trivacancy after two $4 s$ electrons of Fe transferred into $3 d$ orbitals and sharing two $2 p$ electrons from neighbouring $\mathrm{C}$ atoms.

Adsorbate opens the band gap of graphene with btrivacancy. Fe adatom has formed covalent bonds with adjacent $\mathrm{C}$ atoms in the b-trivacancy region, and the number of free electrons is reduced, resulting in the opening of the energy gap.

The band structures for Fe@BTV are shown in Figure 4. It could be found that an energy gap is opened by trapping $\mathrm{Fe}$ to the b-trivacancy and the energy gap is larger in Figure 4(a) than that in Figure 4(b). The value of energy gap is 0.32 and 0.25 for (a) and (b), respectively.

\section{Conclusions}

In conclusion, density functional theory calculations have been performed to investigate the interaction of $\mathrm{Fe}$ adatom with bridged-trivacancy in graphene. The graphene with btrivacancy trapping Fe atom has one stable structure, namely, Fe@BTV. The adsorption of Fe atom can open a finite energy gap in both r-trivacancy and b-trivacancy. The band gap of Fe@BTV is $0.32 \mathrm{eV}$, larger than the value of Fe@RTV $(0.1 \mathrm{eV})$, which is close to the value for developing graphene-based 


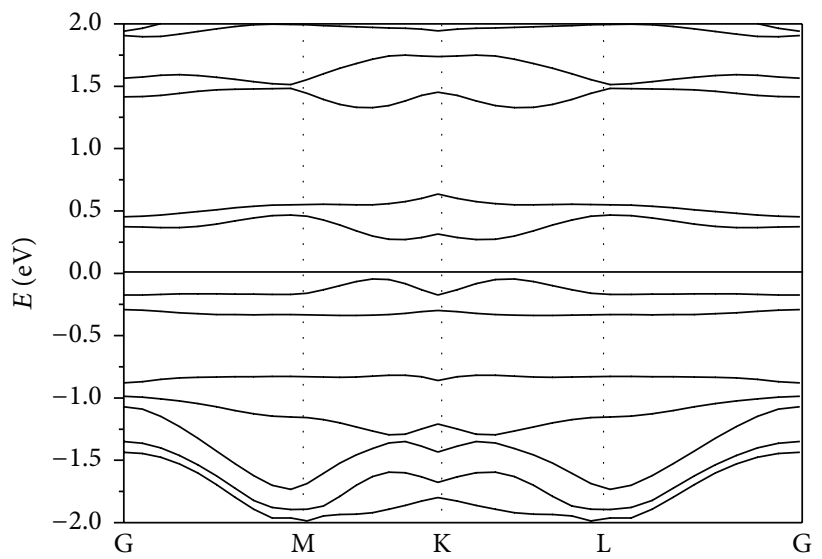

(a)

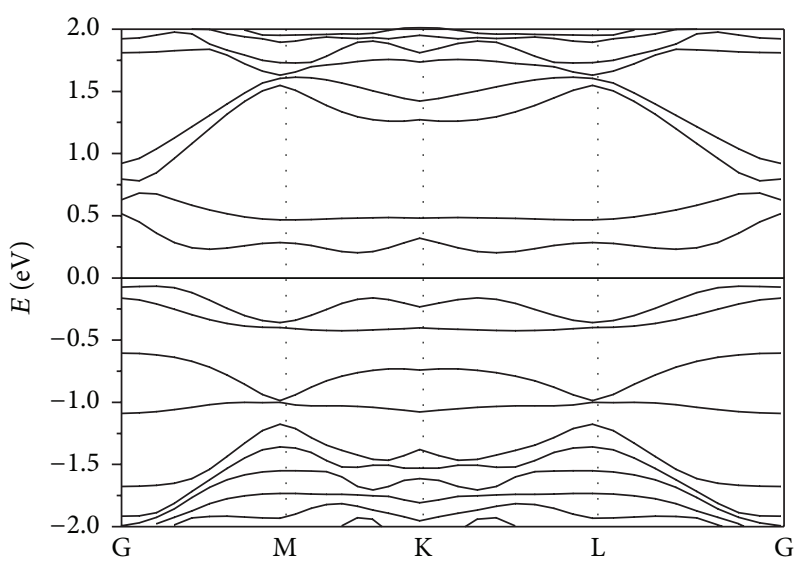

(b)

FIGURE 4: Band structures of Fe@BTV complex. (a) Optimized $5 \times 5$ graphene supercell with b-trivacancy trapping Fe. (b) Optimized $6 \times 6$ graphene supercell with b-trivacancy trapping Fe.

electronics. Fe adatom has formed covalent bonds with adjacent $\mathrm{C}$ atoms in the $\mathrm{b}$-trivacancy region, and the number of free electrons is reduced, resulting in the opening of the energy gap. The findings might provide a promising scheme for gap opening of graphene for industrial semiconductor applications and the photocatalytic technology.

\section{Conflict of Interests}

The authors declare that there is no conflict of interests regarding the publication of this paper.

\section{Acknowledgments}

The authors thank R. Yu for discussions. This work was supported by National Basic Research Program of China (2011CB606406) and the Fundamental Research Funds for the Central Universities (TP-A3: 06108170). This work used the resources of Shanghai Supercomputer Center.

\section{References}

[1] S. Das, M. Kim, J. Lee, and W. Choi, "Synthesis, properties, and applications of 2-D materials: a comprehensive review," Critical Reviews in Solid State and Materials Sciences, vol. 39, no. 4, pp. 231-252, 2014.

[2] A. K. Geim and K. S. Novoselov, "The rise of graphene," Nature Materials, vol. 6, no. 3, pp. 183-191, 2007.

[3] O. V. Yazyev and L. Helm, "Defect-induced magnetism in graphene," Physical Review B-Condensed Matter and Materials Physics, vol. 75, no. 12, Article ID 125408, 2007.

[4] A. H. Castro Neto, F. Guinea, N. M. R. Peres, K. S. Novoselov, and A. K. Geim, "The electronic properties of graphene," Reviews of Modern Physics, vol. 81, article 109, 2009.

[5] Y. Mao, J. Yuan, and J. Zhong, "Density functional calculation of transition metal adatom adsorption on graphene," Journal of Physics Condensed Matter, vol. 20, no. 11, Article ID 115209, 2008.
[6] Y. Zhang, S. Talapatra, S. Kar, R. Vajtai, S. K. Nayak, and P. M. Ajayan, "First-principles study of defect-induced magnetism in carbon," Physical Review Letters, vol. 99, Article ID 107201, 2007.

[7] M. Topsakal, E. Aktürk, H. Sevinçli, and S. Ciraci, "Firstprinciples approach to monitoring the band gap and magnetic state of a graphene nanoribbon via its vacancies," Physical Review B, vol. 78, Article ID 235435, 2008.

[8] T. Susi, J. Kotakoski, D. Kepaptsoglou et al., "Silicon-carbon bond inversions driven by $60-\mathrm{keV}$ electrons in graphene," Physical Review Letters, vol. 113, no. 11, Article ID 115501, 2014.

[9] K. S. Novoselov, Z. Jiang, Y. Zhang et al., "Room-temperature quantum hall effect in graphene," Science, vol. 315, no. 5817, p. 1379, 2007.

[10] Y. Zhang, Y.-W. Tan, H. L. Stormer, and P. Kim, "Experimental observation of the quantum Hall effect and Berry's phase in graphene," Nature, vol. 438, no. 7065, pp. 201-204, 2005.

[11] J. Ding, Z. Qiao, W. Feng, Y. Yao, and Q. Niu, "Engineering quantum anomalous/valley Hall states in graphene via metalatom adsorption: an ab-initio study," Physical Review B, vol. 84, Article ID 195444, 2011.

[12] D. Xiao, W. Yao, and Q. Niu, "Valley-contrasting physics in graphene: magnetic moment and topological transport," Physical Review Letters, vol. 99, Article ID 236809, 2007.

[13] F. Guinea, M. I. Katsnelson, and A. K. Geim, "Energy gaps and a zero-field quantum Hall effect in graphene by strain engineering," Nature Physics, vol. 6, pp. 30-33, 2010.

[14] C. L. Kane and E. J. Mele, " $Z_{2}$ topological order and the quantum spin hall effect," Physical Review Letters, vol. 95, no. 14, Article ID 146802, 2005.

[15] D. W. Boukhvalov and M. I. Katsnelson, "Tuning the gap in bilayer graphene using chemical functionalization: density functional calculations," Physical Review B, vol. 78, no. 8, Article ID 085413, 2008.

[16] M. Hammouri, S. K. Jha, and I. Vasiliev, "First-principles study of graphene and carbon nanotubes functionalized with benzyne," The Journal of Physical Chemistry C, vol. 119, no. 32, pp. 18719-18728, 2015.

[17] A. J. Yan and M. Y. Chou, "Oxidation functional groups on graphene: structural and electronic properties," Physical Review $B$, vol. 82, Article ID 125403, 2010. 
[18] J. B. Oostinga, H. B. Heersche, X. Liu, A. F. Morpurgo, and L. M. K. Vandersypen, "Gate-induced insulating state in bilayer graphene devices," Nature Materials, vol. 7, pp. 151-157, 2008.

[19] K. T. Chan, J. B. Neaton, and M. L. Cohen, "First-principles study of metal adatom adsorption on graphene," Physical Review B, vol. 77, no. 23, Article ID 235430, 2008.

[20] Z. He, K. He, A. W. Robertson et al., "Atomic structure and dynamics of metal dopant pairs in graphene," Nano Letters, vol. 14, no. 7, pp. 3766-3772, 2014.

[21] X. Q. Dai, J. H. Zhao, M. H. Xie, Y. N. Tang, Y. H. Li, and B. Zhao, "First-principle study of magnetism induced by vacancies in graphene," The European Physical Journal B-Condensed Matter and Complex Systems, vol. 80, no. 3, pp. 343-349, 2011.

[22] A. V. Krasheninnikov and R. M. Nieminen, "Attractive interaction between transition-metal atom impurities and vacancies in graphene: a first-principles study," Theoretical Chemistry Accounts, vol. 129, no. 3-5, pp. 625-630, 2011.

[23] O. Cretu, A. V. Krasheninnikov, J. A. Rodríguez-Manzo, L. Sun, R. M. Nieminen, and F. Banhart, "Migration and localization of metal atoms on strained graphene," Physical Review Letters, vol. 105, Article ID 196102, 2010.

[24] S. Haldar, B. S. Pujari, S. Bhandary et al., " $\mathrm{Fe}_{n}(n=1-6)$ clusters chemisorbed on vacancy defects in graphene: stability, spin-dipole moment, and magnetic anisotropy," Physical Review B, vol. 89, no. 20, Article ID 205411, 2014.

[25] A. V. Krasheninnikov, P. O. Lehtinen, A. S. Foster, P. Pyykkö, and R. M. Nieminen, "Embedding transition-metal atoms in graphene: structure, bonding, and magnetism," Physical Review Letters, vol. 102, no. 12, Article ID 126807, 4 pages, 2009.

[26] G. Kim, S. Jhi, S. Lim, and N. Park, "Effect of vacancy defects in graphene on metal anchoring and hydrogen adsorption," Applied Physics Letters, vol. 94, Article ID 173102, 2009.

[27] T. Eelbo, M. Waśniowska, P. Thakur et al., "Adatoms and clusters of $3 \mathrm{~d}$ transition metals on graphene: electronic and magnetic configurations," Physical Review Letters, vol. 110, no. 13, Article ID 136804, 2013.

[28] Y. Han, G.-X. Ge, J.-G. Wan, J.-J. Zhao, F.-Q. Song, and G.-H. Wang, "Predicted giant magnetic anisotropy energy of highly stable Ir dimer on single-vacancy graphene," Physical Review B, vol. 87, Article ID 155408, 2013.

[29] A. W. Robertson, B. Montanari, K. He et al., "Dynamics of single Fe atoms in graphene vacancies," Nano Letters, vol. 13, no. 4, pp. 1468-1475, 2013.

[30] A. V. Krasheninnikov, P. O. Lehtinen, A. S. Foster, P. Pyykkö, and R. M. Nieminen, "Embedding transition-metal atoms in graphene: structure, bonding, and magnetism," Physical Review Letters, vol. 102, no. 12, Article ID 126807, 2009.

[31] J. A. Rodríguez-Manzo, O. Cretu, and F. Banhart, "Trapping of metal atoms in vacancies of carbon nanotubes and graphene," ACS Nano, vol. 4, no. 6, pp. 3422-3428, 2010.

[32] K. Zhou, Y. Zhu, X. Yang, and C. Li, "One-pot preparation of graphene $/ \mathrm{Fe}_{3} \mathrm{O}_{4}$ composites by a solvothermal reaction," New Journal of Chemistry, vol. 34, no. 12, pp. 2950-2955, 2010.

[33] G. Wang, T. Liua, X. Xiea, Z. Renb, J. Baic, and H. Wang, "Structure and electrochemical performance of $\mathrm{Fe}_{3} \mathrm{O}_{4}$ /graphene nanocomposite as anode material for lithium-ion batteries," Materials Chemistry and Physics, vol. 128, no. 3, pp. 336-340, 2011.

[34] W. Fan, W. Gao, C. Zhang, W. W. Tjiu, J. Pan, and T. Liu, "Hybridization of graphene sheets and carbon-coated $\mathrm{Fe}_{3} \mathrm{O}_{4}$ nanoparticles as a synergistic adsorbent of organic dyes," Journal of Materials Chemistry, vol. 22, no. 48, pp. 25108-25115, 2012.
[35] A. W. Robertson, G.-D. Lee, K. He, E. Yoon, A. I. Kirkland, and J. H. Warner, "The role of the bridging atom in stabilizing odd numbered graphene vacancies," Nano Letters, vol. 14, no. 7, pp. 3972-3980, 2014.

[36] H. Wang, Q. Wang, Y. Cheng et al., "Doping monolayer graphene with single atom substitutions," Nano Letters, vol. 12, no. 1, pp. 141-144, 2012.

[37] P. E. Blöchl, "Projector augmented-wave method," Physical Review B, vol. 50, Article ID 17953, 1994.

[38] G. Kresse and J. Furthmuller, "Efficiency of ab-initio total energy calculations for metals and semiconductors using a plane-wave basis set," Computational Materials Science, vol. 6, no. 1, pp. 15-50, 1996.

[39] J. P. Perdew, K. Burke, and M. Ernzerhof, "Generalized gradient approximation made simple," Physical Review Letters, vol. 77, article 3865, 1996. 

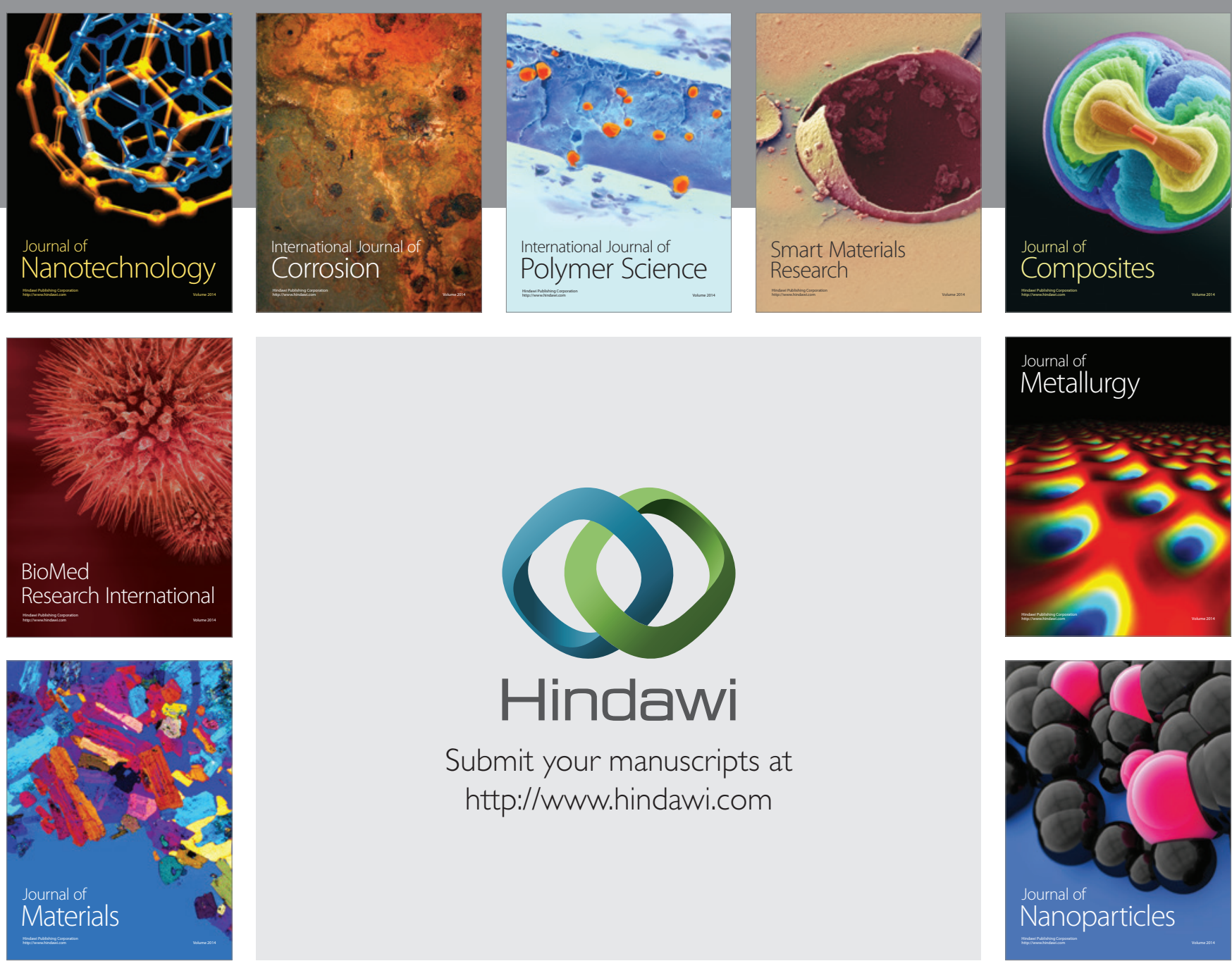

\section{Hindawi}

Submit your manuscripts at

http://www.hindawi.com

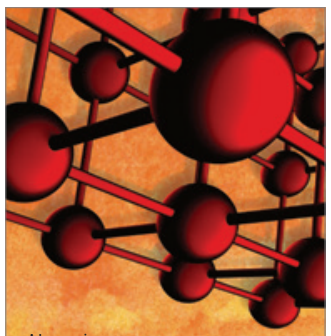

Materials Science and Engineering
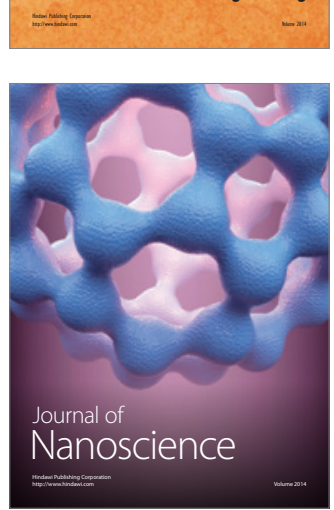
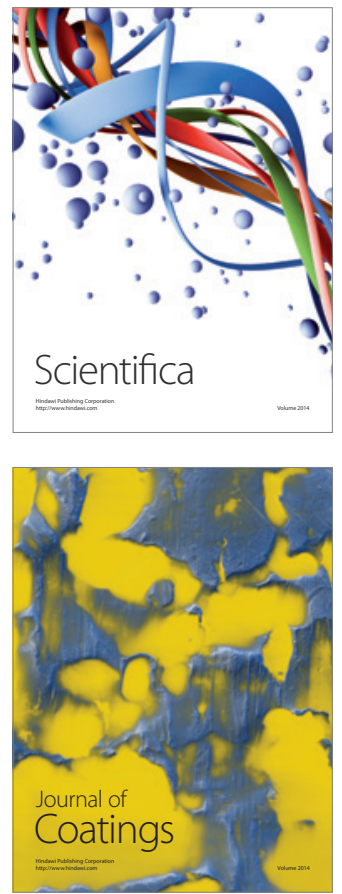
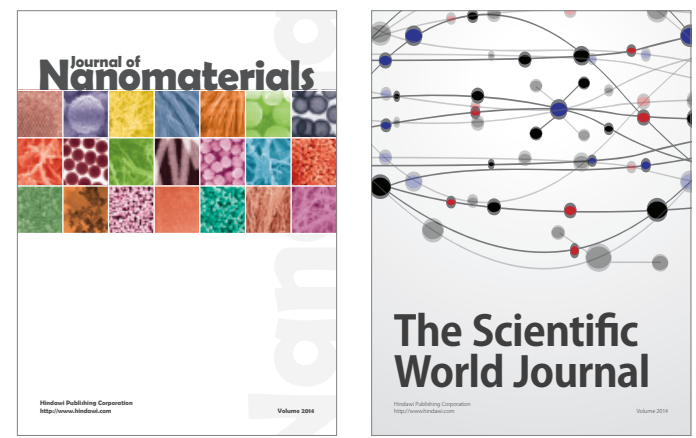

The Scientific World Journal
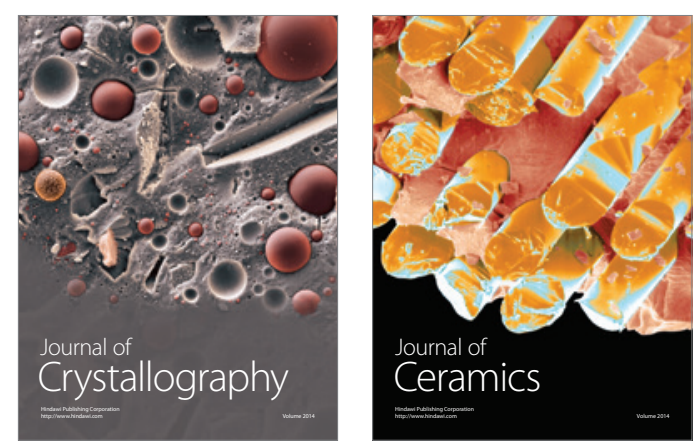
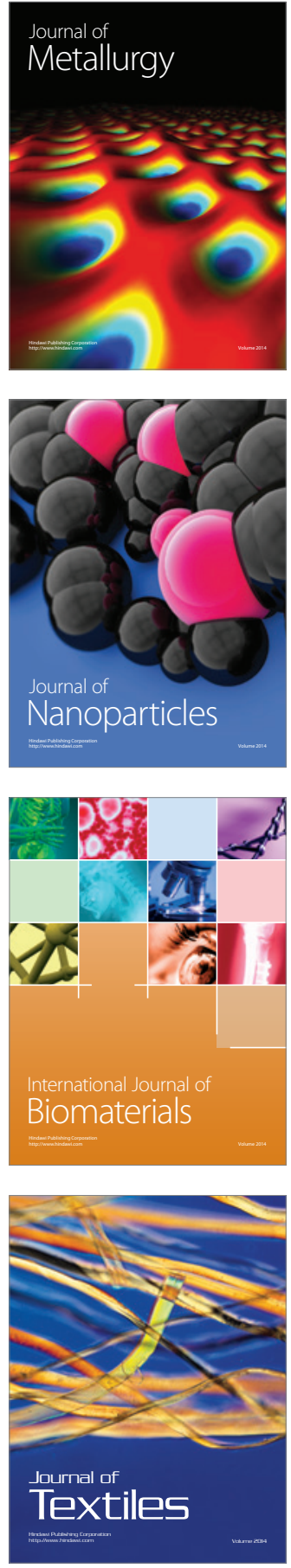